\title{
New insight into the nitrogen metabolism in APB
}

\author{
Bitong ZHu, Chungui ZHAO, SUPING YANG ${ }^{1}$
}

${ }^{1}$ Department of Bioengineering and Biotechnology, Huaqiao University, Xiamen 361021, China.

It is great importance of controlling nitrogen pollution by revealing the vital factors affecting bacterial nitrogen cycle. Anaerobic phototrophic bacteria (APB) are considered as tool for the study of photosynthesis, $\mathrm{N}$ and $\mathrm{S}$ cycle. A marine APB (Marichromatium gracile YL28) isolated from intertidal sediment of mangrove could utilized high concentration of nitrite as the sole nitrogen source for cell growth. It possesses the diverse key genes involved in denitrification (DN), assimilatory nitrate reduction (ANR) and fermentative nitrate reduction (DNRA) in genome. However, the coordination mechanisms of $\mathrm{N}$ cycles remain unclear. This study further verified the funtions of key genes involved in nitrogen cycle at expression levels by qPCR, and revealed the coordinating mechanism in response to different environmental changes. The results showed that (1) the expression level of $n r f A$ (DNRA) were changed with oxgygen and nitrate concentrations, oxygen had significant effect on $n r f A$ expression than light, indicating that DNRA was active and regulated by environmental factors; (2) narI and napA involved in DN and ANR were significantly expressed in the light $(\mathrm{p}<0.05)$, whereas $n r f A$ was little affected $(\mathrm{p}<0.05)$, indicating that light positively regulated the DN and ANR activity rather than dark. (3) the expresion levels of key genes anaerobically were higher than that in aerobic condition, indicating that elevated oxygen had negative effect on nitrogen cycle of YL28, whreas the elevated nitrate promoted the gene expression $(\mathrm{p}<0.05)$. (4) Oxygen regulated negatively the the expression of narl, norB (DN) and nirA(ANR), but the genes were still expressed, indicating that DN and ANR could facultatively perform nitrogen cycle, which was favourable for survival in mangrove intertidal special ecosystem. So far, DN was only found in PNSB but not in PSB, ANR was only reported in a APB strain, DNRA was not found in APB. Our study was the first time indicated that DNRA paythway was presented in APB. DN was found in PSB. NrfA were tightly regulated in a lower oxygen level. Nitrate had a positive regulatory on the three pathways. It was reported that denitrification might be mediated by napA. nirA and $n r f \mathrm{~A}$ were also mediated by the napA. Light has significant positive contribution to the $\mathrm{DN}$ and nir A gene expression. except for $n r f \mathrm{~A}$.

Acknowledgements

This work was supported by National Marine Public Industry Research (No. 201505026), by Natural Science Foundation of Fujian Province (No. 2018J01049). 